\title{
Extracorporeal membrane oxygenation as treatment of graft failure after heart transplantation
}

\author{
Ciro Mastroianni ${ }^{1,2}$, Antonio Nenna ${ }^{1}$, Guillaume Lebreton ${ }^{2}$, Cosimo D'Alessandro $^{2}$, Salvatore Matteo Greco', \\ Mario Lusini ${ }^{1}$, Pascal Leprince ${ }^{2}$, Massimo Chello ${ }^{1}$ \\ ${ }^{1}$ Department of Cardiovascular Surgery, Università Campus Bio-Medico di Roma, Rome, Italy; ${ }^{2}$ Department of Cardiac Surgery, Hôpital \\ Universitaire Pitié-Salpêtrière, Paris, France \\ Correspondence to: Ciro Mastroianni, MD, PhD. Department of Cardiovascular Surgery, Università Campus Bio-Medico di Roma, Via Alvaro del \\ Portillo 200, 00128 Rome, Italy. Email: c.mastroianni@unicampus.it.
}

\begin{abstract}
Heart transplantation (HTx) is a valuable option in eligible patients with end-stage heart failure. The most significant complication in the immediate post-operative period is early graft failure (EGF), with a mean incidence of $20-25 \%$. EGF is a major risk factor for death and accounts for $40-50 \%$ of early mortality after HTx. Despite the use of inotropes, EGF may persist and require temporary mechanical circulatory support. Extracorporeal membrane oxygenation (ECMO) has been investigated over the years and has proved to be a reliable strategy in patients with EGF after HTx. This study aims to review the contemporary literature on this topic. Considering short-term outcomes, $45-80 \%$ of patients were discharged alive from hospital. Duration of support is variable, with a mean duration of 4-8 days. Cannulation strategy and device selection have no differences with respect to short-term outcomes. The main causes of death are multi-organ failure, bleeding, heart failure, stroke and sepsis. Considering long-term outcomes, ECMO survivors appear to have similar survival rates to HTx patients who did not experience EGF. Also, ECMO-treated EGF, among survivors, has no detrimental effect for graft function. In conclusion, ECMO is a reliable therapeutic option to support patients with severe graft failure after HTx, providing adequate support with either central or peripheral arteriovenous cannulation. Further studies will be needed to establish the correct threshold for ECMO support and to provide long-term results.
\end{abstract}

Keywords: Extracorporeal membrane oxygenation (ECMO); graft failure; heart transplantation (HTx)

Submitted Dec 06, 2018. Accepted for publication Dec 17, 2018.

doi: $10.21037 /$ acs.2018.12.08

View this article at: http://dx.doi.org/10.21037/acs.2018.12.08

\section{Extracorporeal membrane oxygenation (ECMO) as treatment of graft failure after heart transplantation (HTx): a "simple" mechanical solution to a multifactorial problem}

HTx is a valuable option in eligible patients with end-stage heart failure, secondary to cardiomyopathy, failed palliation of congenital heart disease or acquired cardiac disease (1-4). One of the most significant complications in the immediate post-operative period is early graft failure (EGF), with a mean incidence of 20-25\% (1,3-5). EGF can result from long ischemic time, increased donor age, inadequate myocardial preservation at time of procurement, increased pulmonary vascular resistances, hyperacute rejection, or poor adaptation of the graft to the recipient's hemodynamic environment $(1,5)$. EGF is a major risk factor for death and accounts for $40-50 \%$ of early mortality after HTx $(4,6,7)$, with a 4.5 -fold increased risk of mortality compared to HTx patients without EGF $(15 \%$ vs. $60 \%)(5,7,8)$ and represents the most common cause of in-hospital mortality after $\operatorname{HTx}(3,5)$, with a negative impact on early and late outcomes (5). The absence of EGF is associated with better long-term survival (94\% at 1 year and $81 \%$ at 5 years) compared to patients with severe EGF (survival: $36 \%$ at 1 year and $28 \%$ at 5 years) (7).

Despite the use of inotropes (e.g., dobutamine, epinephrine) and pulmonary vasodilators (e.g., inhaled nitric oxide, prostacyclin), EGF may persist and require 
temporary mechanical circulatory support (TMCS), which is usually performed using extracorporeal membrane oxygenation (ECMO) or ventricular assist devices (VAD). ECMO has been investigated over the years and has proved to be a reliable strategy in patients with EGF after HTx. This study aims to review the contemporary literature on this topic, to enlighten new avenues for future research.

\section{Clinical outcomes}

The main results of studies evaluating ECMO as treatment of EGF after HTx are summarized in Table 1, indicating the short-term and long-term outcomes, where available. All studies are retrospective, as no randomized trials have been recently performed on this topic. Considering the short-term outcomes, $45-80 \%$ of patients could be defined as "ECMO survivors", since they are discharged alive from hospital. Duration of support is variable, with a mean duration of 4-8 days. A central or a peripheral cannulation strategy and device selection are determined by surgeon's preference and center's practice, with no differences in short-term outcomes. The main causes of death are multiorgan failure, bleeding, heart failure, stroke and sepsis. Considering long-term outcomes, ECMO survivors appear to have similar survival rates to HTx patients who did not experience EGF. Also, ECMO-treated EGF, among survivors, has no detrimental effect for graft function.

\section{Diagnosis of EGF and its risk factors}

According to the last ISHLT consensus statement (18), EGF is classified as primary when the triggering factor is unknown and secondary when the cause can be determined, such as hyperacute rejection, pulmonary hypertension or surgical complication. The diagnosis of EGF should be made within 24 hours after HTx procedure and EGF is graded as mild, moderate or severe based on specified criteria $(7,18)$, such as ejection fraction, cardiac index, pulmonary capillary wedge pressure, mean arterial pressure, inotropic score and intra-aortic balloon pump (IABP) use. The etiology of EGF after HTx is multifactorial and involves donor-recipient mismatching, inadequate donor heart preservation, use of marginal donor hearts and $\mathrm{ABO}-$ incompatible $\mathrm{HTx}$ (3). Inadequate donor heart preservation, due to long ischemia time and other pre-harvest issues, such as long "downtime", high dose inotropic agent titration and preoperative anoxic insults from sepsis, drowning, or asphyxia, are known predictors of EGF after HTx $(1,9)$.
A recent study concluded that the predictive factors for moderate-to-severe EGF occurrence are preoperative trans-pulmonary gradient $>12 \mathrm{mmHg}$ [odds ratio (OR) 5.2], a preoperative inotropic score $>10$ (OR 8.5) and preoperative ECMO support (OR 4.2) (7). Another study investigated donor [age $>60$ years with OR 2.1, preoperative mechanical circulatory support (MCS) with OR 2.6] and recipient features (mean norepinephrine dose with OR 2.0, trauma as the cause of death with OR 2.4, left ventricular ejection fraction $<55 \%$ with OR 2.7 , ischemic time with OR 1.01) as predictive risk factors for EGF (11). With increasingly liberal criteria for donor organ acceptance, the incidence of EGF may be expected to increase in the next years, and therefore future efforts should try to define the optimal EGF treatment to reduce morbidity and mortality. TMCS can be utilized to achieve hemodynamic stability for adjuvant therapy administration, such as immunological treatments (3), with the hope for eventual cardiac recovery.

Along with the beneficial effects of ECMO in postcardiotomy low cardiac output syndrome (LCOS) (1), ECMO has become a reasonable and effective option for EGF in children and adults after HTx (1). ECMO for graft failure after HTx appears to have an improved survival rate compared to patients experiencing a cardiac arrest and placed on ECMO as a bridge to transplantation or compared to other etiologies (55\% vs. 30-40\%) $(1,2,9,19)$, indicating that the donor's heart is more likely to recover after cardiac failure than a failing ventricle in need of transplantation, and that acute decompensation following HTx is often a treatable process. Also, ECMO provides a full circulatory support with minimal surgical trauma, avoiding end-organ damage and allowing both ventricles to rest and recover $(5,20)$.

\section{Central versus peripheral cannulation}

ECMO support can be deployed via peripheral or central cannulation, with previous studies indicating that shortterm survival is not dependent on the type of cannulation (5). Evidence from the literature indicates that the risk of mediastinitis and the incidence of other major morbidities is similar between central and peripheral cannulation, except for an increased risk of vascular complication (e.g., femoral) in peripheral ECMO $(5,12)$. In some patients, however, femoral cannulation cannot provide a satisfactory circulatory support probably because of an insufficient venous drainage or a relatively small diameter of the arterial cannula (5); also, peripheral ECMO use is daunted by the issues of pulmonary edema and might require strategies to 


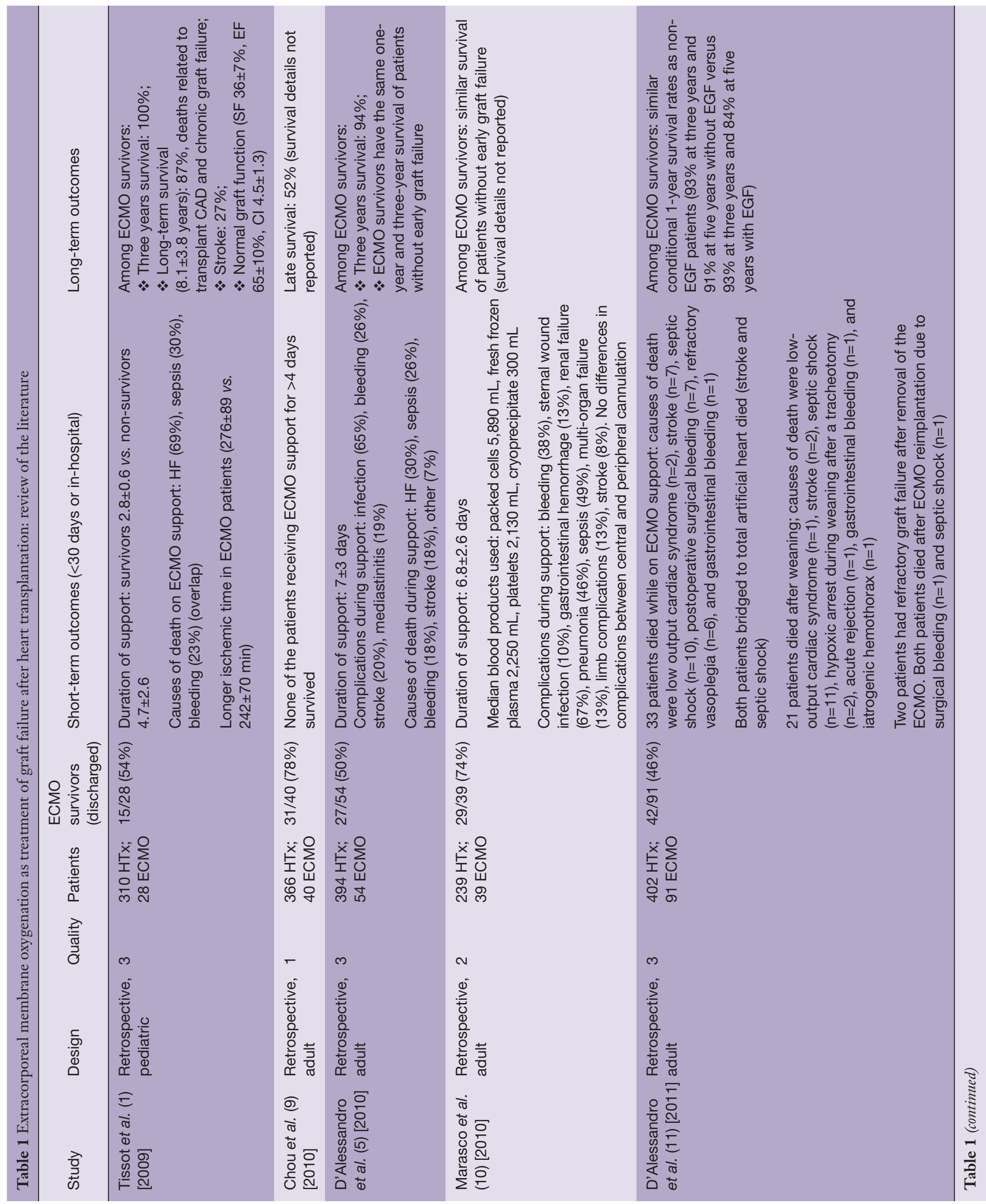




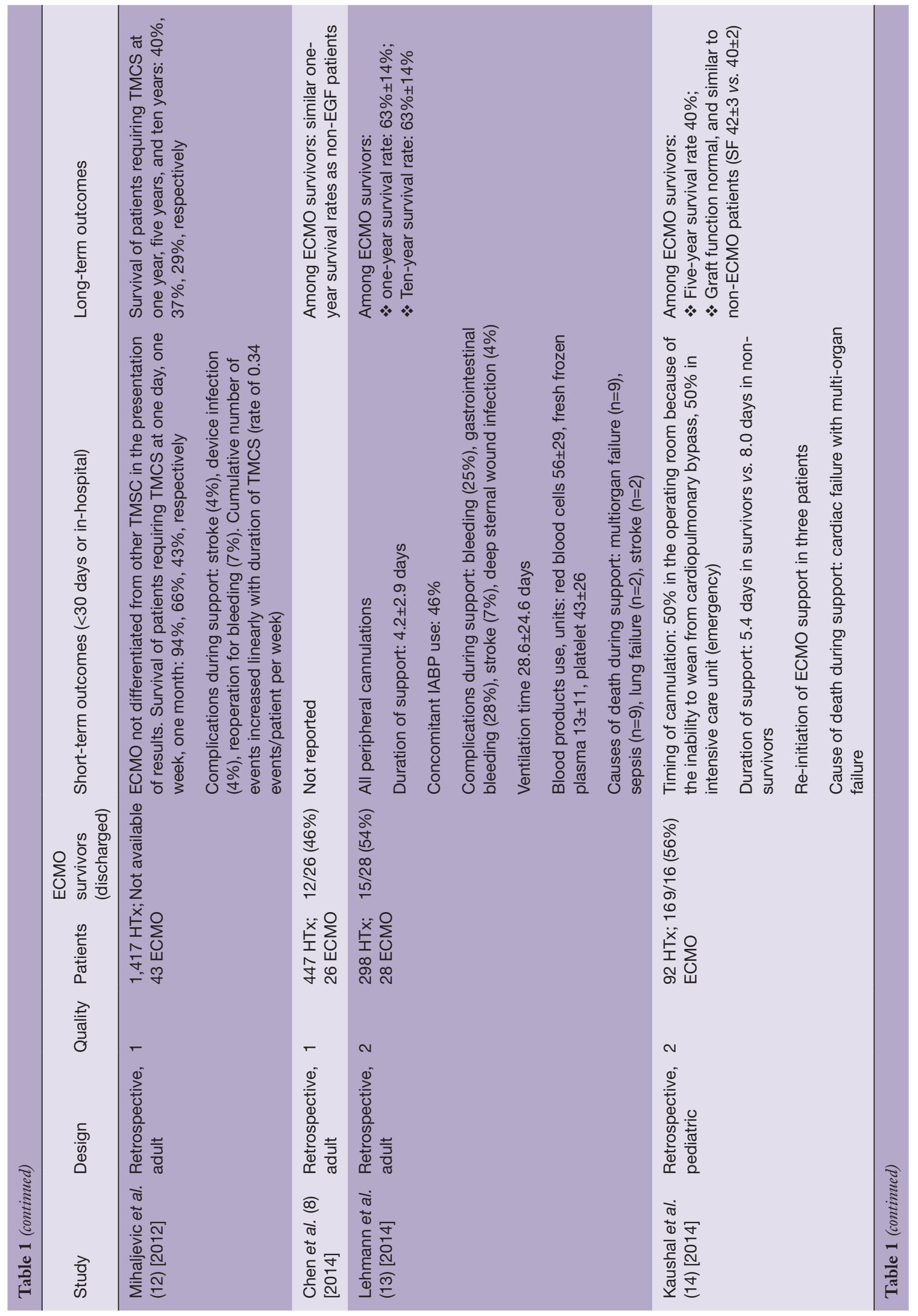




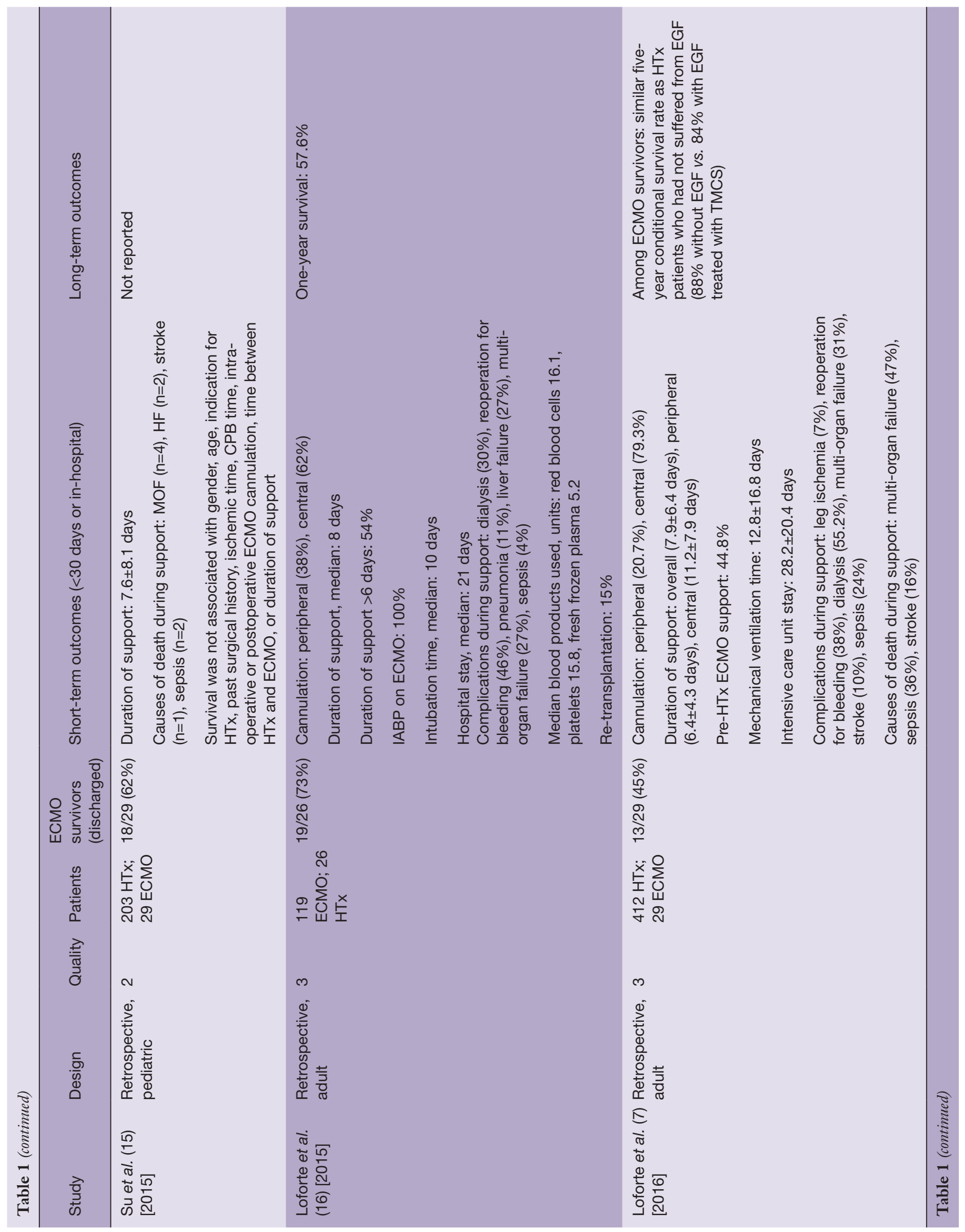




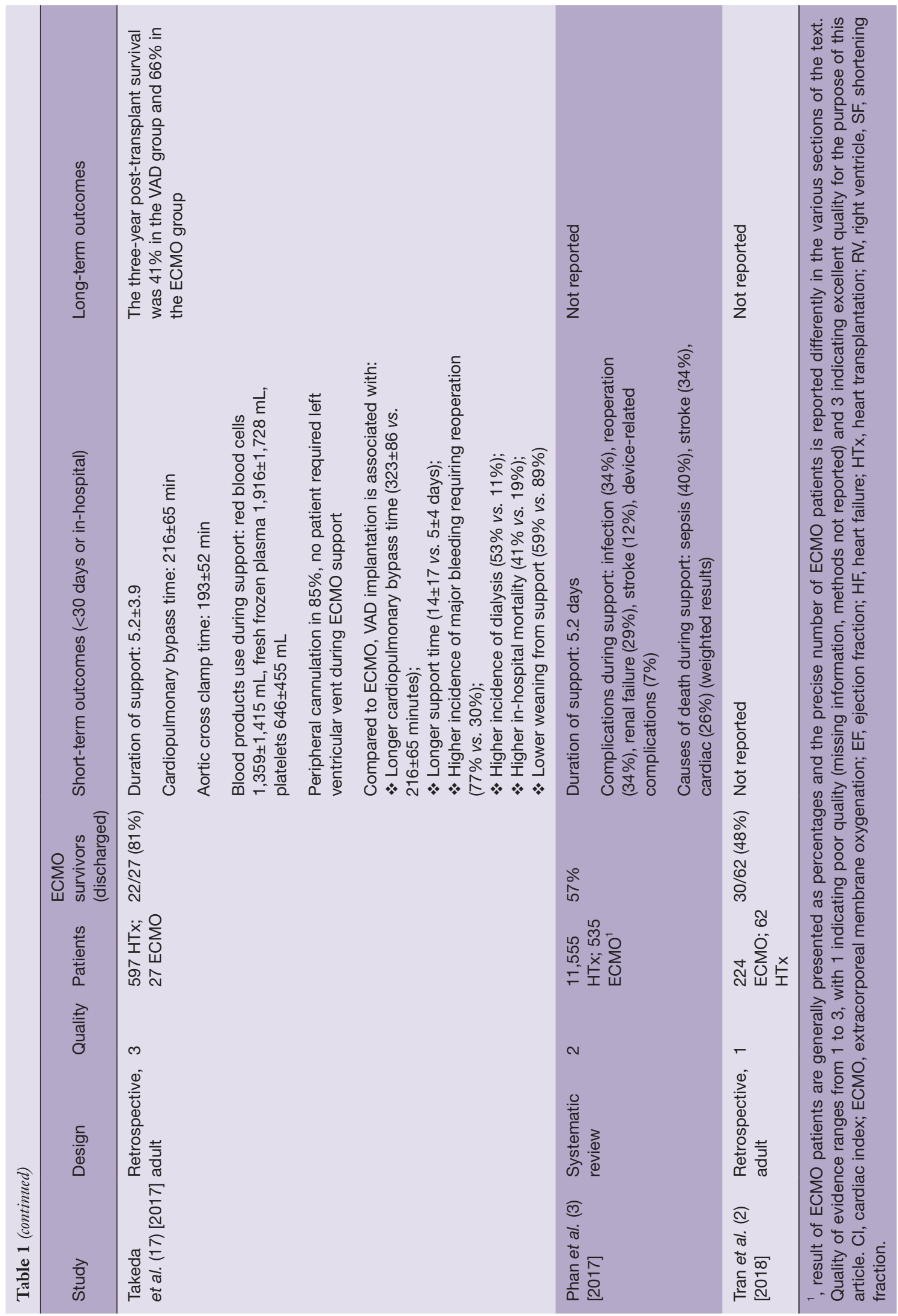


vent the left ventricle $(4,6)$.

In brief, each technique has advantages and disadvantages (10), as the choice of central cannulas allows better flow, antegrade flow through the arch without admixing with deoxygenated blood, avoiding lower limb vascular complications; on the other hand, the drawback is the need to reopen the sternum to remove the cannulas. The peripheral cannulas allow the sternum to be closed without having to go back in to that surgical field. However, it is more difficult to achieve high flows, which may be necessary in larger patients, (retrograde flow is delivered up the descending aorta with the risk of admixing in the arch) and there is always the risk of lower limb vascular complications (10). The actual policy in many centers is ECMO support with central cannulation, unless the patient is on peripheral ECMO preoperatively, or if the implantation is performed on an emergency basis (12). In most cases, the decision is based on surgeon preference $(10,12)$. Future studies will clarify this issue and will aid in the establishment of new guidelines.

\section{Duration of ECMO support}

ECMO usage is costly with high short-term mortality, which has not significantly improved over the past decades (2). Patient selection remains crucial and early predictors of unsuccessful ECMO therapy should be investigated. As is to be expected, a longer duration of ECMO support is significantly associated with poorer outcomes. In general, most ECMO survivors are decannulated within four days from the initiation of support (1). However, other studies support the notion that graft survival may take longer to recover and should not be confined to a predetermined time limit (14), noticing no differences in ability of the graft to recover even up to eight days post-support on ECMO (14).

A longer duration of support, despite being encouraged and ethically required, especially in younger patients, is associated with increased risk of sepsis, bleeding and neurologic sequelae and a tailored risk/benefit ratio should be evaluated. An alternative solution to circumvent the daunting problems associated with longer ECMO support could be the evaluation for re-transplantation or a transition to a VAD if the patient could not be weaned from ECMO support in 4-5 days (1). Emergency re-transplantation has been attempted in the past, but it carried an unacceptable operative mortality and therefore has been abandoned as a "one size fits all" procedure $(3,5)$. The shortage of donors and the increased risk of a redo procedure requires that re-transplantation should be reserved for extremely wellselected patients (12). In particular, patients with severe neurologic injury, irreversible organ injury, hyperacute rejection and infections do not appear to be appropriate candidates for re-transplantation.

\section{Neurologic complications}

ECMO-related complications are common, with an incidence of about $30 \%$, with limb ischemia being the most frequent and neurological events being the most feared (2). As far as neurological complications are concerned, emergent cannulation is associated with a greater risk of neurological complications, as shown by the analysis of patients with neurological sequelae (1). Anticipating graft failure with elective cannulation before hemodynamic crash might be a wise strategy to improve neurological outcomes.

\section{Long-term outcomes}

Long-term survival of ECMO survivors after EGF is excellent and comparable to non-ECMO patients, as survival rates among ECMO survivors is $>90 \%$ at 3 years and $>80 \%$ after a mean follow-up of 8 years $(1,5)$. In another study, inhospital weaned and survived patients after IABP and ECMO treatment for moderate-to-severe EGF had a similar five-year conditional survival rate compared to transplant patients who had not suffered from EGF (88\% without EGF versus $84 \%$ with EGF treated with TMCS devices) (7). Graft function appears to be not influenced by the use of ECMO, as shown by shortening fraction, ejection fraction and cardiac index $(1,14)$. Interestingly, for those ECMO patients who survived, the graft recovered to normal function, which suggests that the stunned myocardium might have the ability to recover and possibly to regenerate the damaged myocardium (14). EGF does not appear to affect late outcomes, with patients experiencing similar graft function and survival as other transplanted patients $(5,12)$. Therefore, these patients have to overcome the initial critical phase, after which they can live a lifespan comparable to transplanted patients who did not experience EGF. Strengthened intensive care and identification of the ideal bridge to recovery is vital to the success of this therapy $(3,12)$.

\section{Alternatives to ECMO support: VAD}

Although ECMO can provide adequate support, it has limitations, including limited LV unloading, limited time 
of support and risks of thromboembolic and vascular complications (4). MCS can also be achieved with shortterm VAD. Veno-arterial ECMO may potentially be more effective than VADs in patients with EGF after HTx as it can be implanted quicker and more easily at bedside $(3,14)$. Also, compared with VADs, ECMO is more versatile in configuration, providing circulatory and respiratory support, has fewer thromboembolic complications and is associated with reduced costs (10,21). Moreover, the direct ventriculotomy involved with VAD implantation can increase the risk of hemorrhage $(3,22)$. A recent retrospective study compared VAD and ECMO in the case of EGF after HTx (17), indicating that patients who received VAD were more likely to have longer cardiopulmonary bypass times, longer support times ( $\mathrm{P}=0.011)$, higher incidence of major bleeding requiring chest re-exploration $(\mathrm{P}=0.0047)$ and higher incidence of renal failure requiring renal replacement therapy $(\mathrm{P}=0.0045)$ after surgery. Overall hospital mortality was $27 \%$; inhospital mortality for VAD and ECMO patients were $41 \%$ and $19 \%$, respectively $(\mathrm{P}=0.16)(17)$. The 3 -year posttransplant survival in VAD and ECMO groups were $41 \%$ and $66 \%$, respectively $(\mathrm{P}=0.13)(17)$. Therefore, this study is the first to indicate that for severe primary graft dysfunction (PGD), support with ECMO appears to result in better clinical outcomes than VAD support (17).

However, percutaneous implantation of right VAD has become available (3), despite many anatomic limitations. Also, more aggressive strategies, such as biventricular support including a durable VAD or a total artificial heart are currently reported in small studies (4). Those minimally invasive or aggressive approaches remain a promising alternative to the traditional open approach and should be further investigated in future studies. At present, there is insufficient high-quality evidence (randomized studies or propensity score matched studies) to prefer ECMO over VAD and the optimal modality of TMCS following HTx should be determined by the surgeon and institutional experience (18), depending on the extent and severity of myocardial dysfunction, and considering the presence or absence of associated respiratory insufficiency $(18,21)$.

\section{Special issues: pediatric population}

Patients with congenital heart disease undergoing HTx in the recent era have become more complex, with a greater number of prior cardiac operations and complex vascular reconstructions, requiring prolonged intra-operative preparation (23). Pediatric heart recipients requiring ECMO support for EGF are younger compared with the overall transplantation population, and longer ischemic time is a crucial risk factor for graft dysfunction (1). Duration of ECMO did not adversely impact graft function and is an acceptable therapy for infants after HTx for LCOS (14). A recent article evaluated the risk factors for requiring ECMO support in pediatric patients, which were namely ventilation (OR 2.7), total cardiopulmonary bypass time (OR 1.7) and preoperative inotropes (OR 4.7) (14). ECMO is associated with increased risk of reoperation for bleeding $(80 \% \mathrm{vs}$. $25 \%$ in non-ECMO patients), whereas other complications such as mediastinitis, seizures, sepsis, dialysis and stroke do not seem to be significantly influenced (14).

\section{Clinical implications}

An implementation of ECMO support should be considered early on the verge of inevitable cardiac allograft failure (13). If HTx failure is thought to be temporary and reversible, it is imperative to prevent secondary organ damage, cardiopulmonary resuscitation, or profound metabolic acidosis prior to starting ECMO therapy to allow for successful recovery on ECMO support (13). The use of ECMO is a valuable bridge to recovery strategy in HTx patients with EGF-related low cardiac output. The long-term outcomes of hospital survivors are comparable with that of HTx patients without ECMO therapy, and therefore, ECMO use is an efficient and widely available mechanical support technique to overcome severe cardiac HTx dysfunction. However, because of the high morbidity, ECMO indication has to be considered only after a thorough individual risk assessment. Because of the high device-related morbidity, further efforts have to be made to develop less traumatic devices, allowing fast and easy applicable devices for temporary circulatory support (13).

\section{Limitations}

It is important to acknowledge that there is large heterogeneity in the patient population included in the cited studies. Differences in patient selection criteria, surgical technique, donor choice and definition of EGF (3) are major limitations, which prevents quantitative analysis of such data.

\section{Conclusions}

ECMO is a reliable therapeutic option to support patients 
with severe graft failure after cardiac transplantation, providing adequate support with either central or peripheral arteriovenous cannulation with excellent survival-todischarge rates. Shorter duration of support is an important factor for early survival, although ECMO support should be maintained for a reasonable yet undetermined time period as "latecomer's hearts" might have a postponed recovery. Late mortality and graft function seem not to be affected by post-transplant ECMO among survivors. Further studies will be needed to establish the correct threshold for ECMO support and to provide long-term results.

\section{Acknowledgements}

None.

\section{Footnote}

Conflicts of Interest: The authors have no conflicts of interest to declare.

\section{References}

1. Tissot C, Buckvold S, Phelps CM, et al. Outcome of extracorporeal membrane oxygenation for early primary graft failure after pediatric heart transplantation. J Am Coll Cardiol 2009;54:730-7.

2. Tran BG, De La Cruz K, Grant S, et al. Temporary Venoarterial Extracorporeal Membrane Oxygenation: Ten-Year Experience at a Cardiac Transplant Center. J Intensive Care Med 2018;33:288-95.

3. Phan K, Luc JGY, Xu J, et al. Utilization and Outcomes of Temporary Mechanical Circulatory Support for Graft Dysfunction After Heart Transplantation. ASAIO J 2017;63:695-703.

4. Bermudez CA, McMullan DM. Extracorporeal life support in preoperative and postoperative heart transplant management. Ann Transl Med 2017;5:398.

5. D'Alessandro C, Aubert S, Golmard JL, et al. Extracorporeal membrane oxygenation temporary support for early graft failure after cardiac transplantation. Eur J Cardiothorac Surg 2010;37:343-9.

6. Lund LH, Edwards LB, Kucheryavaya AY, et al. The Registry of the International Society for Heart and Lung Transplantation: Thirty-second Official Adult Heart Transplantation Report--2015; Focus Theme: Early Graft Failure. J Heart Lung Transplant 2015;34:1244-54.

7. Loforte A, Murana G, Cefarelli M, et al. Role of Intra-
Aortic Balloon Pump and Extracorporeal Membrane Oxygenation in Early Graft Failure After Cardiac Transplantation. Artif Organs 2016;40:E136-45.

8. Chen JW, Chen YS, Chi NH, et al. Risk factors and prognosis of patients with primary graft failure after heart transplantation: an Asian center experience. Transplant Proc 2014;46:914-9.

9. Chou NK, Chi NH, Wu IW, et al. Extracoporeal membrane oxygenation to rescue cardiopulmonary failure after heart transplantation: a single-center experience. Transplant Proc 2010;42:943-5.

10. Marasco SF, Vale M, Pellegrino V, et al. Extracorporeal membrane oxygenation in primary graft failure after heart transplantation. Ann Thorac Surg 2010;90:1541-6.

11. D'Alessandro C, Golmard JL, Barreda E, et al. Predictive risk factors for primary graft failure requiring temporary extra-corporeal membrane oxygenation support after cardiac transplantation in adults. Eur J Cardiothorac Surg 2011;40:962-9.

12. Mihaljevic T, Jarrett CM, Gonzalez-Stawinski G, et al. Mechanical circulatory support after heart transplantation. Eur J Cardiothorac Surg 2012;41:200-6; discussion 206.

13. Lehmann S, Uhlemann M, Etz CD, et al. Extracorporeal membrane oxygenation: experience in acute graft failure after heart transplantation. Clin Transplant 2014;28:789-96.

14. Kaushal S, Matthews KL, Garcia X, et al. A multicenter study of primary graft failure after infant heart transplantation: impact of extracorporeal membrane oxygenation on outcomes. Pediatr Transplant 2014;18:72-8.

15. Su JA, Kelly RB, Grogan T, et al. Extracorporeal membrane oxygenation support after pediatric orthotopic heart transplantation. Pediatr Transplant 2015;19:68-75.

16. Loforte A, Pilato E, Martin Suarez S, et al. RotaFlow and CentriMag extracorporeal membrane oxygenation support systems as treatment strategies for refractory cardiogenic shock. J Card Surg 2015;30:201-8.

17. Takeda K, Li B, Garan AR, et al. Improved outcomes from extracorporeal membrane oxygenation versus ventricular assist device temporary support of primary graft dysfunction in heart transplant. J Heart Lung Transplant 2017;36:650-6.

18. Kobashigawa J, Zuckermann A, Macdonald P, et al. Report from a consensus conference on primary graft dysfunction after cardiac transplantation. J Heart Lung Transplant 2014;33:327-40.

19. Fenton KN, Webber SA, Danford DA, et al. Long- 
term survival after pediatric cardiac transplantation and postoperative ECMO support. Ann Thorac Surg 2003;76:843-6; discussion 847.

20. Loforte A, Marinelli G, Musumeci F, et al. Extracorporeal membrane oxygenation support in refractory cardiogenic shock: treatment strategies and analysis of risk factors. Artif Organs 2014;38:E129-41.

21. Urban M, Szarszoi O, Pirk J, et al. What is the optimal mode of mechanical support in transplanted patients with acute graft failure? Interact Cardiovasc Thorac Surg

Cite this article as: Mastroianni C, Nenna A, Lebreton G, D'Alessandro C, Greco SM, Lusini M, Leprince P, Chello M. Extracorporeal membrane oxygenation as treatment of graft failure after heart transplantation. Ann Cardiothorac Surg 2019;8(1):99-108. doi: 10.21037/acs.2018.12.08
2013;16:517-9.

22. Thomas HL, Dronavalli VB, Parameshwar J, et al. Incidence and outcome of Levitronix CentriMag support as rescue therapy for early cardiac allograft failure: a United Kingdom national study. Eur J Cardiothorac Surg 2011;40:1348-54.

23. Shi WY, Saxena P, Yong MS, et al. Increasing Complexity of Heart Transplantation in Patients With Congenital Heart Disease. Semin Thorac Cardiovasc Surg 2016;28:487-97. 